Z Epileptol 2009 $\cdot 22: 108-108$ DOI 10.1007/s10309-009-0034-0

Online publiziert: 17. April 2009

(c) Springer-Verlag 2009

\section{Arbeitsgemeinschaft für Epilepsie Franken (AGEF)}

\section{Versorgungskonzept zur flächendeckenden Epilepsieversorgung in Franken}

Die Arbeitsgemeinschaft für Epilepsie Franken (AGEF), gegründet 2008, hat sich als wichtige Aufgabe die Entwicklung und Realisierung eines flächendeckenden Epilepsieversorgungskonzeptes in Franken zum Ziel gesetzt. Durch die Vernetzung professioneller und sektorübergreifender Kompetenz in der Region und die Nutzung von Synergien soll eine optimale Patientenversorgung aus medizinischer und ökonomischer Sicht erreicht werden. Alle Beteiligten in dieser Gemeinschaft verstehen sich als Partner in der Patientenversorgung.

In einem ersten Schritt stellt die AGEF regionale Versorgungsschwerpunkte verschiedener Einrichtungen zur Epilepsieversorgung vor. Dadurch wollen wir eine zielgerichtete Patientenzuweisung von niedergelassenen Neurologen, Hausärzten, sozialmedizinischen Stellen sowie internistischen Abteilungen und Altersheimen erreichen.

\section{Kompetenzzentrum Epilepsiezentrum Erlangen (ZEE)}

Leitung: Prof. Dr. med. Hermann Stefan

Leistungsspektrum. Schwer zu diagnostizierende und behandelbare Epilepsien, Zweitmeinung, medikamentöse und operative Behandlung, Stimulationsverfahren (Nervus-vagus-, Tiefenhirnstimulation etc.), Langzeit-Video-EEG-Monitoring und mobiles Langzeit-EEG-Monitoring (MLE), therapeutisches Monitoring (TISA), präoperative Diagnostik, Magnetenzephalograhie (MEG), computergestützte Testung kognitiver Funktionen (CCETE).

\section{Kompetenzzentrum Neurologische Klinik Bad Neustadt}

Arbeitsgruppe Epilepsie: Dr. med. Tobias Kniess

Leistungsspektrum. Medizinische Rehabilitation von Anfallspatienten, Psychosomatik, vaskuläre Epilepsie, Synkopendiagnose.

\section{Kompetenzzentrum Neurologische Klinik Bezirkskrankenhaus Bayreuth}

Leitung: Prof. Dr. med. Dipl.Psych. Matthias Keidel

Leistungsspektrum. Altersepilepsie, vaskuläre Epilepsie, spezielle epileptische Syndrome (u. a. Alkohol, Drogen, Medikamente etc.), dissoziative Anfälle, Synkopenabklärung; innerhäusiger Verbund mit Gerontopsychiatrie, Psychosomatik, psychiatrisch-heilpädagogischer Bereich und Suchtbereich.

\section{Kompetenzzentrum Neurologische Klinik Krankenhaus Rummelsberg}

Leitung: PD Dr. med. Martin Winterholler

Leistungsspektrum. Mehrfachbehinderung mit Epilepsie, Differenzialdiagnose nächtlicher Anfälle und Parasomnien (Langzeit-Video-EEG-Monitoring mit Polysomnographie verbunden); Schlafmedizin; Betreuung von Patienten mit Stimulationsverfahren; Epilepsiezentrum mit kliniknahen Einrichtungen der Be- hinderten- und Altenhilfe; Berufsbildungswerk für junge Menschen mit Epilepsie (BBW); epileptologische Institutsambulanz.

Alle Interessenten, die eine flächendeckende, verbesserte Versorgungsstruktur für unsere Patienten mittragen möchten, sind herzlich aufgerufen, mit uns Kontakt aufzunehmen.

\section{Kontaktstelle:}

Dipl. Betriebswirtin (FH)

Ingrid Hercksen

Neurologische Universitätsklinik Erlangen

Epilepsiezentrum (ZEE)

Schwabachanlage 6

91054 Erlangen

Tel. 09131-85-44610

Fax: o9x131-85-34122

E-Mail: ingrid.hercksen@uk-erlangen.de Internet: http://www.epilepsiezentrumerlangen.de 